\title{
An Improved Gyrocompass Alignment Method for Large Azimuth Misalignment
}

\author{
Wei Gao, ${ }^{1}$ Baofeng Lu, ${ }^{1}$ Chunyang Yu, ${ }^{1}$ and Haiyu Lan ${ }^{1,2}$ \\ ${ }^{1}$ College of Automation, Harbin Engineering University, Harbin 150001, China \\ ${ }^{2}$ Department of Geomatics Engineering, University of Calgary, Calgary, AB, Canada T2N 1N4 \\ Correspondence should be addressed to Baofeng Lu; lu_bao_feng@163.com
}

Received 3 August 2014; Revised 12 January 2015; Accepted 12 January 2015

Academic Editor: Francesco Braghin

Copyright (c) 2015 Wei Gao et al. This is an open access article distributed under the Creative Commons Attribution License, which permits unrestricted use, distribution, and reproduction in any medium, provided the original work is properly cited.

\begin{abstract}
Due to the impact of the nonlinear factor caused by large azimuth misalignment, the conventional gyrocompass alignment method is hard to favorably meet the requirement of alignment speed under the condition of large azimuth misalignment of INS. In order to solve this problem, an improved gyrocompass alignment method is presented in this paper. The improved method is designed based on the nonlinear model for large azimuth misalignment and performed by opening the azimuth loop. The influence of the nonlinear factor on gyrocompass alignment will be reduced when opening the azimuth loop. Simulation and experimental results show that the initial alignment can be efficiently accomplished through using the improved method in the case of existing large azimuth misalignment, and in the same conditions, the alignment speed of the improved method is faster than that of the conventional one.
\end{abstract}

\section{Introduction}

The initial alignment of inertial navigation systems (INS) is an important process performed prior to normal navigation procedure [1]. It is well known that the initial alignment result of the system is of fundamental importance to the following navigation accuracy [2]. Therefore, many researchers have investigated this topic, mainly concentrated on gyrocompass alignment and optimal estimation techniques. In contrast to the optimal estimation techniques, the former method does not need precise mathematical and noise model [3]. With many years' development, gyrocompass alignment method based on classical control theory is very mature now. In 1961, Cannon firstly presented gyrocompass alignment method for platform INS [4]. After that, gyrocompass alignment is described extensively in the literatures $[5,6]$, including alignment technique and error analysis. In recent years, with the development of strapdown INS, gyrocompass alignment is applied to strapdown INS [1,7-9]. In all the previous works, gyrocompass alignment is usually designed based on the small angle assumption (i.e., less than 5 degrees), and under this situation, the alignment system can then be approximated as linear model in the case of small azimuth misalignment. However, under the condition of large azimuth misalignment, such approximation is invalid, and the alignment system will then be influenced by the nonlinear factor. Then the conventional method is not effective to properly accomplish initial alignment in the case of existing large azimuth misalignment.

Therefore, in this paper a new improved gyrocompass alignment method, which is applicable to the INS that causes large azimuth misalignment, is established based on the nonlinear model. So far, many works are attempted to model large azimuth misalignment, and several models have been provided, such as the nonlinear psi-angle model $[10,11]$, the rotation vector error and quaternion error models [12], and the nonlinear phi-angle model [13]. In this work, the nonlinear psi-angle model is adopted. The improved method proposed in this paper is performed by opening the azimuth loop, and through using this scheme, the nonlinear factors can be regarded as constant inputs, which contain azimuth misalignment information. The estimation of the azimuth misalignment is implemented by using the horizontal velocity signals to estimate those nonlinear factors. At this time, the impact of the nonlinear factor caused by large azimuth misalignment on gyrocompass alignment can be reduced. 


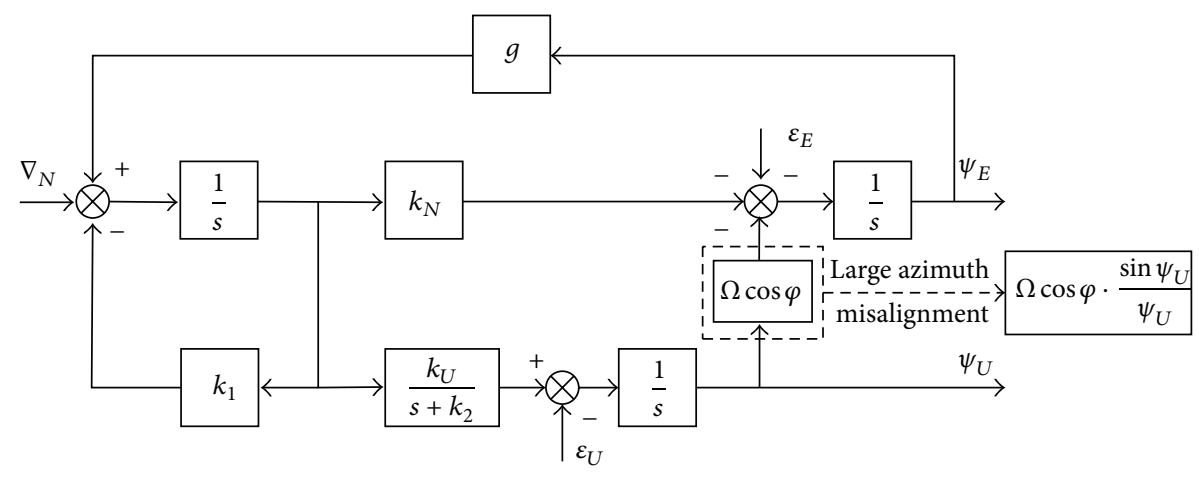

FIGURE 1: Error diagram of conventional gyrocompass alignment.

The remainder of this paper is organized as follows: Section 2 describes the conventional gyrocompass alignment method and then analyzes the existing problem of this method in detail. Section 3 details the improved gyrocompass alignment method, which includes the establishment, operation, and implementation of the improved method. Simulation and experimental results that validate the proposed approach are presented and discussed in Section 4. Finally, conclusions are given in Section 5.

\section{Conventional Gyrocompass Alignment Method}

In literatures $[8,9]$, a fourth-order gyrocompass alignment system was used for INS alignment. It is a higher-order system which has a good performance in the case of small azimuth misalignment. In this paper, the conventional gyrocompass alignment method analyzed here is the fourth-order system (as the representative of the similar methodologies), and the form of this system is simplified. The error diagram of reduced conventional gyrocompass alignment is shown in Figure 1.

In Figure $1, \nabla_{N}$ is the north accelerometer error. $\varepsilon_{E}$ and $\varepsilon_{U}$ are the east and up gyro errors. $\psi_{E}$ and $\psi_{U}$ represent the east level and azimuth misalignments, respectively. $g$ represents the acceleration due to gravity, $\Omega$ represents the earth rate, and $\varphi$ represents the local latitude. $k_{1}, k_{2}, k_{N}$, and $k_{U}$ are the control gains.

According to the Mason gain formula, we can get the characteristic equation of conventional gyrocompass alignment from Figure 1:

$$
\begin{aligned}
\Delta(s)= & s^{2}\left(s+k_{1}\right)\left(s+k_{2}\right)+s\left(s+k_{2}\right) g k_{N} \\
& +g \Omega \cos \varphi k_{U}=0 .
\end{aligned}
$$

In order to ensure the stability of the alignment system, four eigenvalues of (1) are set as $s_{1,2}=-\xi \omega_{n}$ and $s_{3,4}=-\xi \omega_{n} \pm$ $j \sqrt{1-\xi^{2}} \omega_{n}$, where $\xi=0.707$ and $\omega_{n}$ is the natural frequency which is adjustable. Then (1) can be rewritten as

$$
\left(s^{2}+2 \xi \omega_{n} s+\omega_{n}^{2}\right)\left(s+\xi \omega_{n}\right)^{2}=0 .
$$

Comparing (1) with (2), the values of the control gains under the small angle can be obtained:

$$
\begin{aligned}
& k_{1}=k_{2}=2 \xi \omega_{n}, \\
& k_{N}=\frac{\omega_{n}^{2}\left(1+\xi^{2}\right)}{g}, \\
& k_{U}=\frac{\xi^{2} \omega_{n}^{4}}{g \Omega \cos \varphi} .
\end{aligned}
$$

In the knowledge of a classical control theory, it is obvious that the alignment speed of gyrocompass alignment is determined by the eigenvalues. Worth noting is that the four eigenvalues of the conventional gyrocompass alignment system can be given any value desired by appropriate choice of the control gains. In other words, the alignment speed of conventional gyrocompass alignment is determined by choice of the control gains $k_{1}, k_{2}, k_{N}$, and $k_{U}$.

However, if the azimuth misalignment is large, the scale factor $\Omega \cos \varphi$ in Figure 1 will become the nonlinear factor $\Omega \cos \varphi \cdot \sin \psi_{U} / \psi_{U}$. The characteristic equation of conventional gyrocompass alignment becomes

$$
\begin{aligned}
\Delta(s)= & s^{2}\left(s+k_{1}\right)\left(s+k_{2}\right)+s\left(s+k_{2}\right) g k_{N} \\
& +\frac{g \Omega \cos \varphi k_{U}}{k\left(\psi_{U}\right)}=0,
\end{aligned}
$$

where $k\left(\psi_{U}\right)$ denotes the nonlinear factor $\psi_{U} / \sin \psi_{U}$. Then only if the values of the control gains are set as

$$
\begin{aligned}
& k_{1}=k_{2}=2 \xi \omega_{n}, \\
& k_{N}=\frac{\omega_{n}^{2}\left(1+\xi^{2}\right)}{g}, \\
& k_{U}=\frac{k\left(\psi_{U}\right) \cdot \xi^{2} \omega_{n}^{4}}{g \Omega \cos \varphi}
\end{aligned}
$$

can the four eigenvalues of conventional gyrocompass alignment system then be set as $s_{1,2}=-\xi \omega_{n}$ and $s_{3,4}=-\xi \omega_{n} \pm$ $j \sqrt{1-\xi^{2}} \omega_{n}$ (desired values). Since $\psi_{U}$ cannot be obtained, $k\left(\psi_{U}\right)$ is an uncertain factor. Thus, the values of the control 


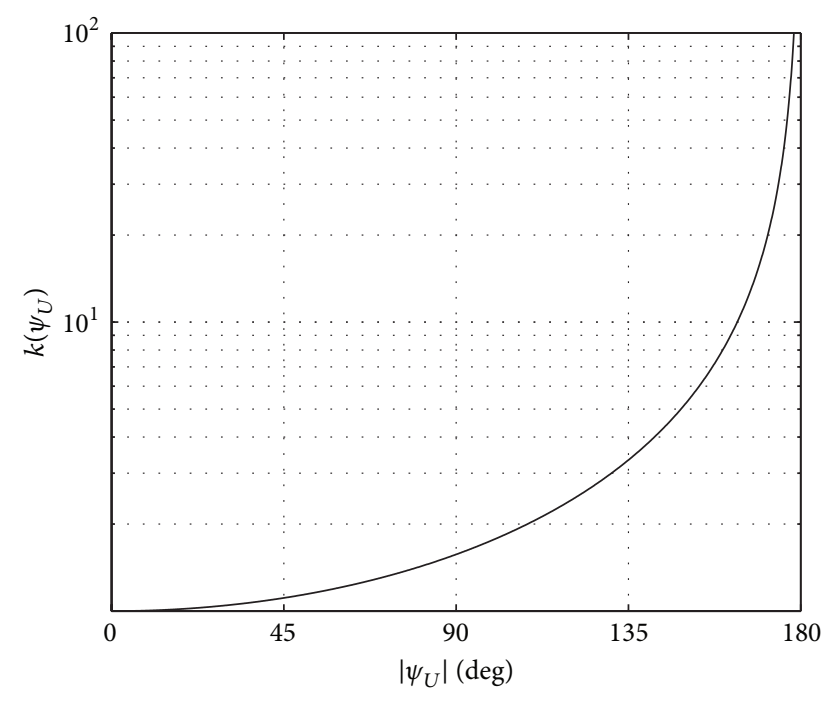

FIGURE 2: Functional relationship between $k\left(\psi_{U}\right)$ and $\left|\psi_{U}\right|$.

gains cannot be set as (7), (8), and (9). In fact, under the large azimuth misalignment, the values of the control gains are still set as (3), (4), and (5). Then four eigenvalues of conventional gyrocompass alignment cannot be set as the desired values. In this case, the conventional method is hard to favorably meet the requirement of alignment speed. The particular reason of this problem also can be expressed by the following equation:

$$
k_{U}=\frac{k_{U}^{\prime}}{k\left(\psi_{U}\right)},
$$

where $k_{U}^{\prime}$ is the theoretical value calculated by (9) and $k_{U}$ is the actual value obtained by (5). From (10), it is clear that the actual value $k_{U}$ adopted by the alignment system is smaller than the theoretical value $k_{U}^{\prime}$, due to the fact that $k\left(\psi_{U}\right) \geq$ 1 (domain: $\psi_{U} \in\left[-180^{\circ}, 180^{\circ}\right]$ ). The functional relationship between $k\left(\psi_{U}\right)$ and $\left|\psi_{U}\right|$ is shown in Figure 2.

It is obvious from Figure 2 that the value of $k\left(\psi_{U}\right)$ is increasing along with the growth of $\left|\psi_{U}\right|$; then the difference between $k_{U}$ and $k_{U}^{\prime}$ will increase together with the growth of $\left|\psi_{U}\right|$. That means the difference between the actual and desired eigenvalues increases along with the growth of $\left|\psi_{U}\right|$; however, the performance of the alignment system will be poor.

\section{Improved Gyrocompass Alignment Method}

Considering the poor performance of conventional gyrocompass alignment under the condition of large azimuth misalignment, an improved gyrocompass alignment method is proposed in this paper to improve the performance of gyrocompass alignment. It is clear that the four eigenvalues of conventional gyrocompass alignment cannot be set as the desired values in the case of large azimuth misalignment, because the uncertain nonlinear factor is included in the characteristic equation. So the key to design the improved method is to reduce the impact of the nonlinear factor on characteristic equation. This problem is solved by opening the azimuth loop; at this time, the influence of the nonlinear factor on characteristic equation disappeared, and the estimation of the azimuth misalignment is implemented by using the horizontal velocity signals to estimate the nonlinear factors.

3.1. The Establishment of the Improved Gyrocompass Alignment Method. In this paper, $i, e, b, n$, and $p$ denote the inertial frame, the earth fixed coordinate frame, the sensor body frame, the navigation frame, and the computed navigation frame, respectively. In this work, we choose the local level geographic coordinate frame as the navigation frame. Under the large azimuth misalignment, the direction cosine matrix (DCM) $C_{n}^{p}$ can be described as follows $[13,14]$ :

$$
\begin{aligned}
& \mathbf{C}_{n}^{p} \\
& =\left[\begin{array}{ccc}
\cos \psi_{U} & \sin \psi_{U} & -\psi_{N} \\
-\sin \psi_{U} & \cos \psi_{U} & \psi_{E} \\
\psi_{N} \cos \psi_{U}+\psi_{E} \sin \psi_{U} & \psi_{N} \sin \psi_{U}-\psi_{E} \cos \psi_{U} & 1
\end{array}\right],
\end{aligned}
$$

where $\psi_{N}$ is the north level misalignment; $\psi_{E}$ and $\psi_{N}$ (level misalignments) are small, and $\psi_{U}$ (azimuth misalignment, the departure of computed north from actual north) is large.

For a quasistationary initial alignment, the average value for velocity will be zero [15]. Then under quasistationary situations, the navigation equations for strapdown INS attitude and velocity can be represented, respectively, as

$$
\begin{aligned}
\dot{\mathbf{C}}_{b}^{p} & =\mathbf{C}_{b}^{p}\left[\boldsymbol{\omega}_{p b}^{b} \times\right], \\
\dot{\mathbf{V}}^{p} & =\mathbf{C}_{b}^{p} \widetilde{\mathbf{f}}^{b}+\mathbf{g}^{n},
\end{aligned}
$$

where $\mathbf{C}_{b}^{p}$ represents the DCM, relating the transformation from $b$ frame to the $p$ frame; $\omega_{p b}^{b}$ is the angular velocity vector of $b$ frame with respect to $p$ frame resolved in $b$ frame; $[\times]$ denotes a skew-symmetric matrix operator; $\mathbf{V}^{p}$ is the computed velocity of strapdown INS; $\widetilde{\mathbf{f}}^{b}$ is the specific force from the accelerometer output; $\mathbf{g}^{n}$ is the gravity vector resolved in $n$ frame. The angular velocity $\omega_{p b}^{b}$ is derived by

$$
\boldsymbol{\omega}_{p b}^{b}=\widetilde{\boldsymbol{\omega}}_{i b}^{b}-\mathbf{C}_{p}^{b} \boldsymbol{\omega}_{i e}^{n}
$$

where $\widetilde{\boldsymbol{\omega}}_{i b}^{b}$ is the angular velocity vector measured by gyros; $\boldsymbol{\omega}_{i e}^{n}$ is the angular velocity vector of $e$ frame with respect to $i$ frame resolved in $n$ frame.

These angular velocity vectors $\widetilde{\boldsymbol{\omega}}_{i b}^{b}, \boldsymbol{\omega}_{i e}^{n}$ and accelerometer output $\widetilde{\mathbf{f}}^{b}$ can be described, respectively, as

$$
\begin{aligned}
\widetilde{\boldsymbol{\omega}}_{i b}^{b} & =\boldsymbol{\omega}_{i b}^{b}+\boldsymbol{\varepsilon}^{b}, \\
\boldsymbol{\omega}_{i e}^{n} & =\left[\begin{array}{lll}
0 & \Omega \cos \varphi & \Omega \sin \varphi
\end{array}\right]^{T}, \\
\widetilde{\mathbf{f}}^{b} & =-\mathbf{g}^{b}+\delta \mathbf{a}^{b}+\nabla^{b},
\end{aligned}
$$

where $\omega_{i b}^{b}$ is the true angular velocity vector, $\boldsymbol{\varepsilon}^{b}$ is the gyro error vector, $\mathbf{g}^{b}$ is the gravity vector resolved in $b$ frame, 
$\delta \mathbf{a}^{b}$ is the disturbing motion vector under quasistationary situations, and $\nabla^{b}$ is the accelerometer error vector.

Substituting (15c) in (13), we obtain

$$
\dot{\mathbf{V}}^{p}=\left(\mathbf{I}-\mathbf{C}_{n}^{p}\right) \mathbf{g}^{n}+\mathbf{C}_{b}^{p} \delta \mathbf{a}^{b}+\mathbf{C}_{b}^{p} \nabla^{b}
$$

Then, from (16), the horizontal components of $\mathbf{V}^{p}$ can be described, respectively, as

$$
\begin{aligned}
& \dot{V}_{E}=-g \psi_{N}+\left(\mathbf{C}_{b}^{p} \delta \mathbf{a}^{b}\right)_{E}+\left(\mathbf{C}_{b}^{p} \nabla^{b}\right)_{E}, \\
& \dot{V}_{N}=g \psi_{E}+\left(\mathbf{C}_{b}^{p} \delta \mathbf{a}^{b}\right)_{N}+\left(\mathbf{C}_{b}^{p} \nabla^{b}\right)_{N},
\end{aligned}
$$

where $V_{E}$ and $V_{N}$ represent the east and north horizontal velocities, respectively; $(\cdot)_{E}$ and $(\cdot)_{N}$ represent the $E$ and $N$ elements of column matrix $(\cdot)$ separately, whose entries from top to bottom are the $E, N$, and $U$ elements.

From (11), (12), and (14), the misalignment equation of strapdown INS under large azimuth misalignment can be represented as $[11,13]$

$$
\dot{\psi}=\left(\mathbf{I}-\mathbf{C}_{n}^{p}\right) \boldsymbol{\omega}_{i e}^{n}-\mathbf{C}_{b}^{p} \boldsymbol{\varepsilon}^{b},
$$

where $\psi$ is the misalignment vector and $\psi=\left[\begin{array}{lll}\psi_{E} & \psi_{N} & \psi_{U}\end{array}\right]^{T}$.

The leveling alignment of the improved method is performed by introducing control angular velocity $\omega_{c}^{p}$ into the calculation of angular velocity $\boldsymbol{\omega}_{p b}^{b}$; then we have $\boldsymbol{\omega}_{p b}^{b}=\widetilde{\boldsymbol{\omega}}_{i b}^{b}-$ $\mathbf{C}_{p}^{b} \boldsymbol{\omega}_{i e}^{n}-\mathbf{C}_{p}^{b} \boldsymbol{\omega}_{c}^{p}$; finally the misalignment equation could be transformed into

$$
\dot{\psi}=\left(\mathbf{I}-\mathbf{C}_{n}^{p}\right) \boldsymbol{\omega}_{i e}^{n}-\mathbf{C}_{b}^{p} \boldsymbol{\varepsilon}^{b}+\boldsymbol{\omega}_{c}^{p},
$$

where $\omega_{c}^{p}=\left[\begin{array}{lll}\omega_{c E}^{p} & \omega_{c N}^{p} & 0\end{array}\right]^{T}$ is the control angular velocity vector. From (19), the horizontal components of $\psi$ can be, respectively, represented as

$$
\begin{aligned}
\dot{\psi}_{E}= & -\sin \psi_{U} \Omega \cos \varphi+\psi_{N} \Omega \sin \varphi-\left(\mathbf{C}_{b}^{p} \boldsymbol{\varepsilon}^{b}\right)_{E}+\omega_{c E}^{p} \\
\dot{\psi}_{N}= & \left(1-\cos \psi_{U}\right) \Omega \cos \varphi-\psi_{E} \Omega \sin \varphi-\left(\mathbf{C}_{b}^{p} \boldsymbol{\varepsilon}^{b}\right)_{N} \\
& +\omega_{c N^{*}}^{p}
\end{aligned}
$$

Because the azimuth misalignment's rate of change is small [10], the approximation $\dot{\psi}_{U}=0$ is admitted; namely, $\psi_{U}$ is a constant value [15].

The azimuth information is obtained by structuring azimuth estimation functions. The block diagram of $\boldsymbol{\omega}_{c}^{p}$ and azimuth estimation functions is shown in Figure 3.

In Figure 3, $k_{a}$ and $k_{b}$ represent the control gains; $G(s)$ and $H(s)$ represent the control networks used for reducing the influence of disturbing motions; $S\left(\psi_{U}\right)$ and $C\left(\psi_{U}\right)$ represent the functions of $\psi_{U}$. From (17), (20), (21), and Figure 3, the error diagrams of improved gyrocompass alignment can be obtained as in Figures 4 and 5.

It can be seen from Figures 4 and 5 that the nonlinear factors $\sin \psi_{U} \Omega \cos \varphi$ and $\left(1-\cos \psi_{U}\right) \Omega \cos \varphi$ both are caused by the large azimuth misalignment and become the constant inputs of the system through opening the azimuth loop. So

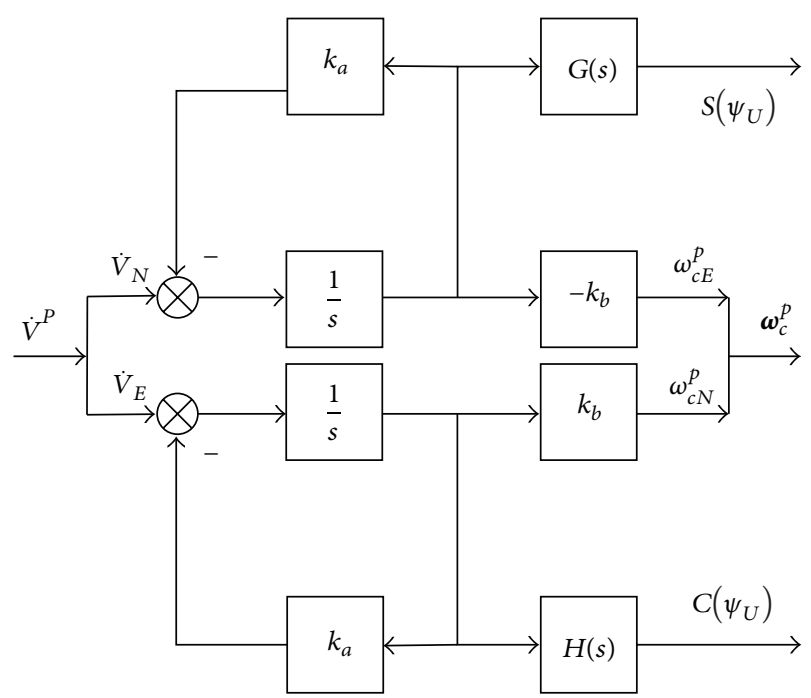

FIgURE 3: Block diagram of $\boldsymbol{\omega}_{c}^{p}$ and azimuth estimation functions.

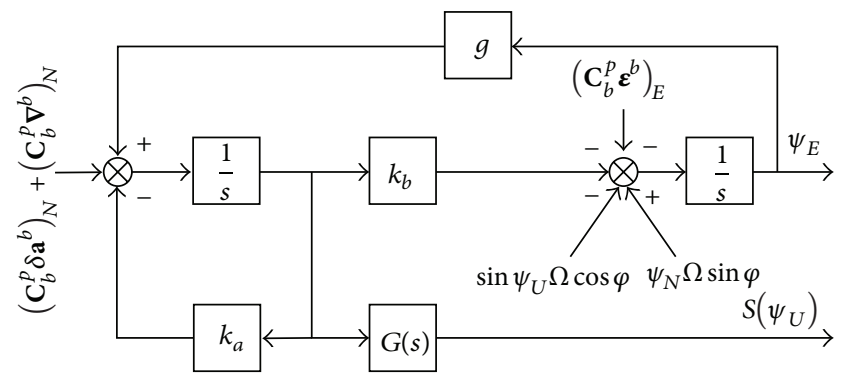

FIGURE 4: Error diagram of improved gyrocompass alignment in north loop.

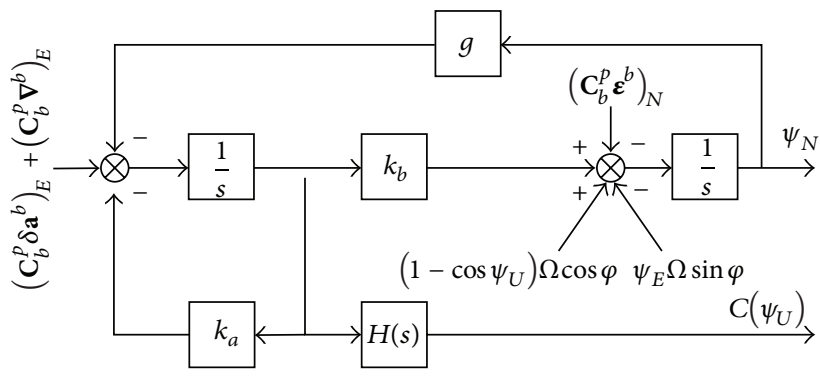

FIGURE 5: Error diagram of improved gyrocompass alignment in east loop.

the nonlinear factors will not be included in the characteristic equation. In addition, these constant inputs contain azimuth misalignment information, and the estimation of the azimuth information can be obtained by $S\left(\psi_{U}\right)$ and $C\left(\psi_{U}\right)$.

3.2. The Operation of the Improved Gyrocompass Alignment Method. In this section, the control gains $k_{a}$ and $k_{b}$ and control networks $G(s)$ and $H(s)$ are provided. Then $S\left(\psi_{U}\right)$ and $C\left(\psi_{U}\right)$ can be obtained, and the azimuth estimation is performed by these two functions. 
Firstly, we provide the values of the control gains $k_{a}$ and $k_{b}$ for improved gyrocompass alignment; the detailed deduction for these control gains can be found in the following.

According to Mason gain formula, we can get the characteristic equation of north loop from Figure 4:

$$
\Delta_{N}(s)=s^{2}+k_{a} s+g k_{b}=0 .
$$

In the same way, from Figure 5, the characteristic equation of east loop can be obtained as follows:

$$
\Delta_{E}(s)=s^{2}+k_{a} s+g k_{b}=0 .
$$

It can be seen from (22) and (23) that the north and east loops have the same characteristic equation. In order to ensure the stability of alignment system, we set two eigenvalues of (22) and (23) as

$$
s_{1,2}=-\xi \omega_{n} \pm j \sqrt{1-\xi^{2}} \omega_{n}
$$

Then the characteristic equation can be rewritten as

$$
\Delta_{N}(s)=\Delta_{E}(s)=s^{2}+2 \xi \omega_{n} s+\omega_{n}^{2}=0 .
$$

Comparing (22) with (25), we can get

$$
\begin{aligned}
k_{a} & =2 \xi \omega_{n}, \\
k_{b} & =\frac{\omega_{n}^{2}}{g} .
\end{aligned}
$$

Secondly, in order to simplify the subsequent analysis, we assume that the inertial sensor errors are basically constant drifts, and the vehicle in which the INS is mounted is totally stopped. Under this assumption, the disturbing motion vector $\delta \mathbf{a}^{b}$ is equal to zero. At this time, the control networks can be set as $G(s)=H(s)=1$, because no disturbing motion is introduced into the alignment system. The design procedure of azimuth estimation is described in the following, and it can be divided into two steps. The first step: the analysis of the relationships between functions $S\left(\psi_{U}\right)$ and $C\left(\psi_{U}\right)$ and azimuth misalignment $\psi_{U}$ is made with the aid of the Laplace transformation. The second step: based on the former analysis, the azimuth estimation equations are provided.

3.2.1. Relationships between Functions $S\left(\psi_{U}\right)$ and $C\left(\psi_{U}\right)$ and Azimuth Misalignment $\psi_{U}$. The response to east level misalignment $\psi_{E}$ and function $S\left(\psi_{U}\right)$ can be written in Laplace form directly from Figure 4 and can be seen as follows (according to the Mason gain formula):

$$
\begin{aligned}
\psi_{E}(s) & \\
= & -\frac{\omega_{n}^{2}}{s^{2}+2 \xi \omega_{n} s+\omega_{n}^{2}} \cdot \frac{\left\{\left(\mathbf{C}_{b}^{p} \nabla^{b}\right)_{N}\right\}(s)}{g} \\
& +\frac{s+2 \xi \omega_{n}}{s^{2}+2 \xi \omega_{n} s+\omega_{n}^{2}} \\
& \cdot\left\{-\sin \psi_{U} \Omega \cos \varphi+\psi_{N} \Omega \sin \varphi-\left(\mathbf{C}_{b}^{p} \boldsymbol{\varepsilon}^{b}\right)_{E}\right\}(s), \\
\left\{S\left(\psi_{U}\right)\right\}(s) & \frac{s}{s^{2}+2 \xi \omega_{n} s+\omega_{n}^{2}} \cdot\left\{\left(\mathbf{C}_{b}^{p} \nabla^{b}\right)_{N}\right\}(s) \\
& +\frac{g}{s^{2}+2 \xi \omega_{n} s+\omega_{n}^{2}} \\
& \cdot\left\{-\sin \psi_{U} \Omega \cos \varphi+\psi_{N} \Omega \sin \varphi-\left(\mathbf{C}_{b}^{p} \boldsymbol{\varepsilon}^{b}\right)_{E}\right\}(s),
\end{aligned}
$$

where $\{\cdot\}(s)$ represents the Laplace transformation of $\{\cdot\}$.

Similarly, from Figure 5, it yields

$$
\begin{aligned}
& \psi_{N}(s)=\frac{\omega_{n}^{2}}{s^{2}+2 \xi \omega_{n} s+\omega_{n}^{2}} \cdot \frac{\left\{\left(\mathbf{C}_{b}^{p} \nabla^{b}\right)_{E}\right\}(s)}{g} \\
& +\frac{s+2 \xi \omega_{n}}{s^{2}+2 \xi \omega_{n} s+\omega_{n}^{2}} \\
& \quad \cdot\left\{\left(1-\cos \psi_{U}\right) \Omega \cos \varphi-\psi_{E} \Omega \sin \varphi-\left(\mathbf{C}_{b}^{p} \boldsymbol{\varepsilon}^{b}\right)_{N}\right\} \\
& \cdot(s), \\
& \left\{C\left(\psi_{U}\right)\right\}(s)=\frac{s}{s^{2}+2 \xi \omega_{n} s+\omega_{n}^{2}} \cdot\left\{\left(\mathbf{C}_{b}^{p} \nabla^{b}\right)_{E}\right\}(s) \\
& -\frac{g}{s^{2}+2 \xi \omega_{n} s+\omega_{n}^{2}} \\
& \cdot\left\{\left(1-\cos \psi_{U}\right) \Omega \cos \varphi-\psi_{E} \Omega \sin \varphi-\left(\mathbf{C}_{b}^{p} \boldsymbol{\varepsilon}^{b}\right)_{N}\right\} \\
& \cdot(s) .
\end{aligned}
$$

Equations (27)-(28) are solved for the steady-state errors by invoking the final value theorem:

$$
\lim _{t \rightarrow \infty} f(t)=\lim _{s \rightarrow 0} s f(s) .
$$


Then we have

$$
\begin{aligned}
& \psi_{E s s}=-\frac{\left(\mathbf{C}_{b}^{p} \nabla^{b}\right)_{N}}{g}+\frac{2 \xi}{\omega_{n}} \cdot\left[-\sin \psi_{U} \Omega \cos \varphi\right. \\
& \left.+\psi_{N s s} \Omega \sin \varphi-\left(\mathbf{C}_{b}^{p} \boldsymbol{\varepsilon}^{b}\right)_{E}\right] \\
& S\left(\psi_{U}\right)_{s s}=\frac{g}{\omega_{n}^{2}} \cdot\left[-\sin \psi_{U} \Omega \cos \varphi+\psi_{N s s} \Omega \sin \varphi\right. \\
& \left.-\left(\mathbf{C}_{b}^{p} \boldsymbol{\varepsilon}^{b}\right)_{E}\right], \\
& \psi_{N s s}=\frac{\left(\mathbf{C}_{b}^{p} \nabla^{b}\right)_{E}}{g}+\frac{2 \xi}{\omega_{n}} \cdot\left[\left(1-\cos \psi_{U}\right) \Omega \cos \varphi\right. \\
& \left.-\psi_{E s s} \Omega \sin \varphi-\left(\mathbf{C}_{b}^{p} \boldsymbol{\varepsilon}^{b}\right)_{N}\right], \\
& C\left(\psi_{U}\right)_{s s}=\frac{g}{\omega_{n}^{2}} \cdot\left[\left(\cos \psi_{U}-1\right) \Omega \cos \varphi+\psi_{E s s} \Omega \sin \varphi\right. \\
& \left.+\left(\mathbf{C}_{b}^{p} \boldsymbol{\varepsilon}^{b}\right)_{N}\right],
\end{aligned}
$$

where $(\cdot)_{\text {ss }}$ represents the steady-state value of $(\cdot)$.

We regard $\sin \psi_{U}, \cos \psi_{U}, \psi_{E s s}$, and $\psi_{N s s}$ as the unknown values; (30) can then be easily solved. Then, the relationships between functions $S\left(\psi_{U}\right)$ and $C\left(\psi_{U}\right)$ and azimuth misalignment $\psi_{U}$ can be obtained:

$$
\begin{aligned}
\sin \psi_{U}= & -\frac{\omega_{n}^{2}}{g \Omega \cos \varphi} \cdot S\left(\psi_{U}\right)_{\mathrm{ss}}-\frac{2 \xi \omega_{n} \tan \varphi}{g} \\
& \cdot C\left(\psi_{U}\right)_{\mathrm{ss}}-\frac{\left(\mathbf{C}_{b}^{p} \boldsymbol{\varepsilon}^{b}\right)_{E}}{\Omega \cos \varphi}+\frac{\left(\mathbf{C}_{b}^{p} \nabla^{b}\right)_{E}}{g} \tan \varphi \\
\cos \psi_{U}= & 1+\frac{\omega_{n}^{2}}{g \Omega \cos \varphi} \cdot C\left(\psi_{U}\right)_{\mathrm{ss}}-\frac{2 \xi \omega_{n} \tan \varphi}{g} \\
& \cdot S\left(\psi_{U}\right)_{\mathrm{ss}}-\frac{\left(\mathbf{C}_{b}^{p} \boldsymbol{\varepsilon}^{b}\right)_{N}}{\Omega \cos \varphi}+\frac{\left(\mathbf{C}_{b}^{p} \nabla^{b}\right)_{N}}{g} \tan \varphi .
\end{aligned}
$$

The detailed derivations of (31) can be found in Appendix A.

3.2.2. The Azimuth Estimation Equations. It is clear that $\sin \psi_{U}$ and $\cos \psi_{U}$ can be supplied exactly by (31) if the accelerometer errors and gyro errors (sensor errors) are known. Since the sensor errors are uncertain, $\sin \psi_{U}$ and $\cos \psi_{U}$ are calculated by the following equations:

$$
\begin{aligned}
\sin \widetilde{\psi}_{U}= & -\frac{\omega_{n}^{2}}{g \Omega \cos \varphi} \cdot S\left(\psi_{U}\right)-\frac{2 \xi \omega_{n} \tan \varphi}{g} \cdot C\left(\psi_{U}\right) \\
\cos \widetilde{\psi}_{U}= & 1+\frac{\omega_{n}^{2}}{g \Omega \cos \varphi} \cdot C\left(\psi_{U}\right)-\frac{2 \xi \omega_{n} \tan \varphi}{g} \\
& \cdot S\left(\psi_{U}\right)
\end{aligned}
$$

where $\sin \widetilde{\psi}_{U}$ and $\cos \widetilde{\psi}_{U}$ are the computed values of $\sin \psi_{U}$ and $\cos \psi_{U}$, respectively. When the improved alignment system is stable, the errors between computed values $\left(\sin \widetilde{\psi}_{U}\right.$ and $\left.\cos \widetilde{\psi}_{U}\right)$ and theoretical values $\left(\sin \psi_{U}\right.$ and $\left.\cos \psi_{U}\right)$ are only determined by sensor errors, and these errors are allowable. Worth noting is that (32) and (33) do not preserve the unit-norm property of the trigonometric function; that is, $\left(\sin \widetilde{\psi}_{U}\right)^{2}+\left(\cos \widetilde{\psi}_{U}\right)^{2} \neq 1$. So normalization of $\sin \widetilde{\psi}_{U}$ and $\cos \widetilde{\psi}_{U}$ should be given, and the simple normalization is provided as follows:

$$
\begin{aligned}
\sin \widehat{\psi}_{U} & =\frac{\sin \widetilde{\psi}_{U}}{\sqrt{\sin ^{2} \widetilde{\psi}_{U}+\cos ^{2} \widetilde{\psi}_{U}}}, \\
\cos \widehat{\psi}_{U} & =\frac{\cos \widetilde{\psi}_{U}}{\sqrt{\sin ^{2} \widetilde{\psi}_{U}+\cos ^{2} \tilde{\psi}_{U}}} .
\end{aligned}
$$

The detailed derivations of (34) can be found in Appendix B.

At this time, the azimuth estimation $\widehat{\psi}_{U}$ can be obtained from (34) and is shown as follows:

$$
\begin{aligned}
& \widehat{\psi}_{U m}=\arcsin \left(\sin \widehat{\psi}_{U}\right) \\
& \widehat{\psi}_{U}= \begin{cases}\widehat{\psi}_{U m} & \cos \widehat{\psi}_{U}>0 \\
180^{\circ}-\widehat{\psi}_{U m} & \cos \widehat{\psi}_{U}<0 \sin \widehat{\psi}_{U}>0 \\
-180^{\circ}-\widehat{\psi}_{U m} & \cos \widehat{\psi}_{U}<0 \sin \widehat{\psi}_{U}<0 .\end{cases}
\end{aligned}
$$

It is obvious from the previous discussion that the azimuth estimation equations consist of (32)-(35).

Finally, we take the disturbing motions into consideration, because the alignment is often performed under quasistationary conditions. The disturbing motions can be generally considered to be sinusoidal $[4,16]$. Then, in order to reduce the influence of the disturbing motions on azimuth estimation, low pass filters need to be added to the system; namely, the control networks $G(s)$ and $H(s)$ should have the capability of restraining disturbance. The design law of the control networks $G(s)$ and $H(s)$ is provided as follows.

(a) The magnification of $G(s)$ and $H(s)$ in low frequency must be equivalent to 1 ; that is, the following equation should be satisfied:

$$
\lim _{s \rightarrow 0} G(s)=\lim _{s \rightarrow 0} H(s)=1 .
$$

The reason for this requirement is that the relationships between functions $S\left(\psi_{U}\right)$ and $C\left(\psi_{U}\right)$ and azimuth misalignment $\psi_{U}$ under quasistationary conditions also can be represented by (31) as the requirement is met.

(b) In high frequency, for the purpose of reducing the influence of disturbing motions, they need be capable of restraining disturbance.

Then, under quasistationary conditions, the azimuth estimation equations also consist of (32)-(35). In this work, the control networks $G(s)$ and $H(s)$ are set as

$$
G(s)=H(s)=\frac{\left(\xi \omega_{n}\right)^{2}}{\left(s+\xi \omega_{n}\right)^{2}} .
$$




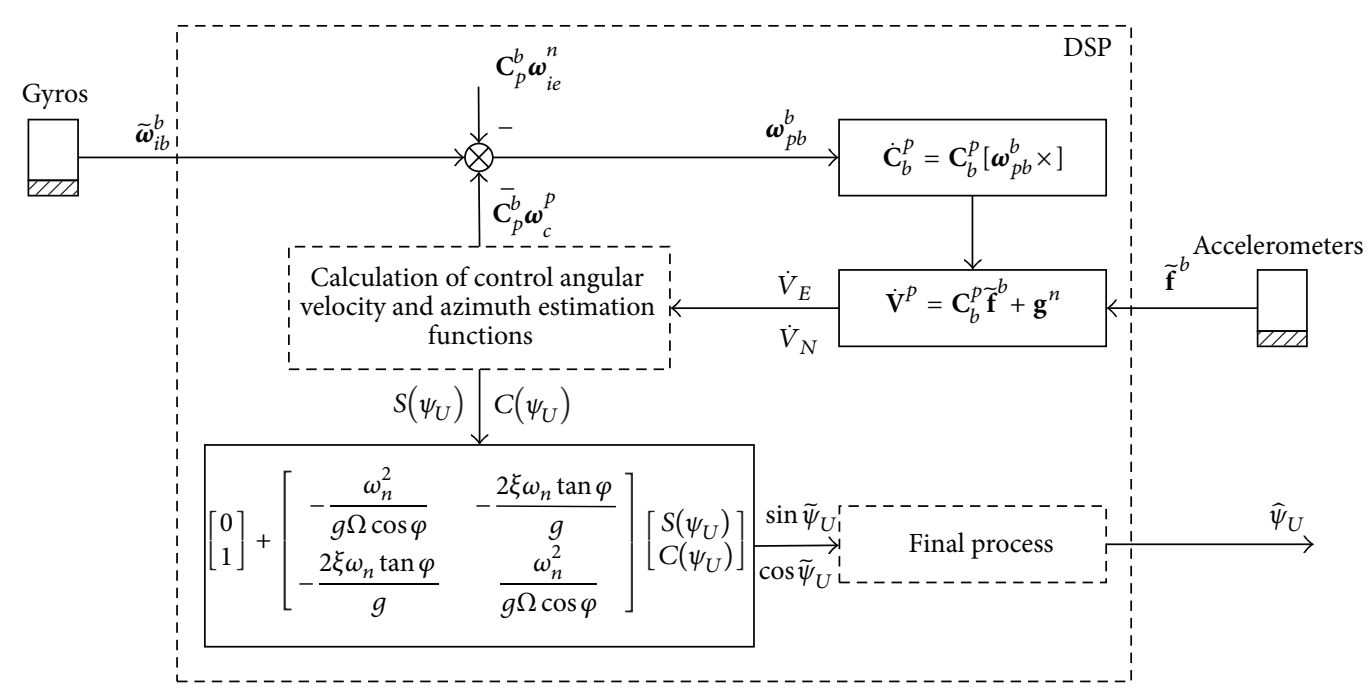

FIgURE 6: Flow chart of improved gyrocompass alignment in real time operating device.

At this time, the denominators of $\left\{S\left(\psi_{U}\right)\right\}(s)$ and $\left\{C\left(\psi_{U}\right)\right\}(s)$ can be set as

$$
\left(s^{2}+2 \xi \omega_{n} s+\omega_{n}^{2}\right)\left(s+\xi \omega_{n}\right)^{2} .
$$

It is obvious from the azimuth estimation equations that the performance of the azimuth alignment is determined by (32) and (33). Since both (32) and (33) consist of $S\left(\psi_{U}\right)$ and $C\left(\psi_{U}\right)$, the four eigenvalues of $\left\{\sin \widetilde{\psi}_{U}\right\}(s)$ and $\left\{\cos \widetilde{\psi}_{U}\right\}(s)$ could be set as $s_{1,2}=-\xi \omega_{n}$ and $s_{3,4}=-\xi \omega_{n} \pm j \sqrt{1-\xi^{2}} \omega_{n}$, without the influence of the nonlinear factors. Therefore, the impact of the nonlinear factor caused by large azimuth misalignment on gyrocompass alignment is reduced, and the performance of the improved gyrocompass alignment will be better than the conventional one.

\subsection{The Implementation of the Improved Gyrocompass Align-} ment Method. In this section, the implementation of the proposed method in real time operating device is discussed, and meanwhile a brief summary of this method is provided. An inertial navigation system implements the proposed alignment method using a cluster of accelerometers to sense the specific force vector components $\widetilde{\mathbf{f}}^{b}$, a triad of gyros to measure the angular velocity $\widetilde{\boldsymbol{\omega}}_{i b}^{b}$, and a digital signal processor (DSP) to perform the alignment algorithm. The direct expression of this implementation can be seen in Figure 6. According to Figure 6, the specific implementation steps of the improved gyrocompass alignment in real time operation device are described as follows.

(a) At the beginning, a preliminary alignment often called coarse alignment is performed, and after that, a rough $\mathrm{DCM} \mathrm{C}_{b}^{p}$ is obtained. The specific coarse alignment method can be found in [15]; the level misalignments are small and azimuth misalignment is usually large.

(b) Secondly, by utilizing the specific force $\widetilde{\mathbf{f}}^{b}$ and DCM $\mathrm{C}_{b}^{p}$, the derivatives of the east and north horizontal velocities $\dot{V}_{E}$ and $\dot{V}_{N}$ can be obtained. Then, according to the calculation method shown in Figure 3, the control velocity $\omega_{c}^{p}$ and azimuth functions $S\left(\psi_{U}\right)$ and $C\left(\psi_{U}\right)$ can be acquired.

(c) On the one hand, the control velocity $\omega_{c}^{p}$ and angular velocity $\widetilde{\boldsymbol{\omega}}_{i b}^{b}$ are used to update the DCM $\mathrm{C}_{b}^{p}$ used for the next calculation step.

(d) On the other hand, the azimuth functions $S\left(\psi_{U}\right)$ and $C\left(\psi_{U}\right)$ are used to compute $\sin \widetilde{\psi}_{U}$ and $\cos \widetilde{\psi}_{U}$. And, after the final process which is achieved by utilizing (34)-(35), the azimuth estimation $\widehat{\psi}_{U}$ is obtained.

(e) Then, steps (b), (c), and (d) are performed repeatedly. Finally, by spending a period of time, an accurate azimuth estimation $\widehat{\psi}_{U}$ can be obtained, and, with the compensation of azimuth misalignment using $\widehat{\psi}_{U}$, an accurate $\mathrm{DCM} \mathrm{C}_{b}^{p}$ can be obtained.

\section{Simulations and Experiments}

In this section, to evaluate the performance of the improved gyrocompass alignment method under the condition of large azimuth misalignment, simulations and experiments are carried out.

4.1. Simulation Results and Analysis. Both the conventional and improved gyrocompass alignment methods are performed during the simulations simultaneously, and the simulations are conducted under the conditions of, respectively, choosing different azimuth misalignments; namely, $\psi_{U}=\left\{50^{\circ}, 100^{\circ}, 145^{\circ},-50^{\circ},-100^{\circ},-145^{\circ}\right\}$. In these simulations, gyro and accelerometer outputs are generated by the strapdown INS simulator; we assume that the vehicle is in disturbing motions caused by sea waves. The initial conditions are presented as follows.

$$
\begin{aligned}
& \text { The constant gyro errors: } \boldsymbol{\varepsilon}^{b}= \\
& {\left[\begin{array}{lll}
0.01^{\circ} / h & 0.01^{\circ} / h & 0.01^{\circ} / h
\end{array}\right]^{T} \text {. }}
\end{aligned}
$$



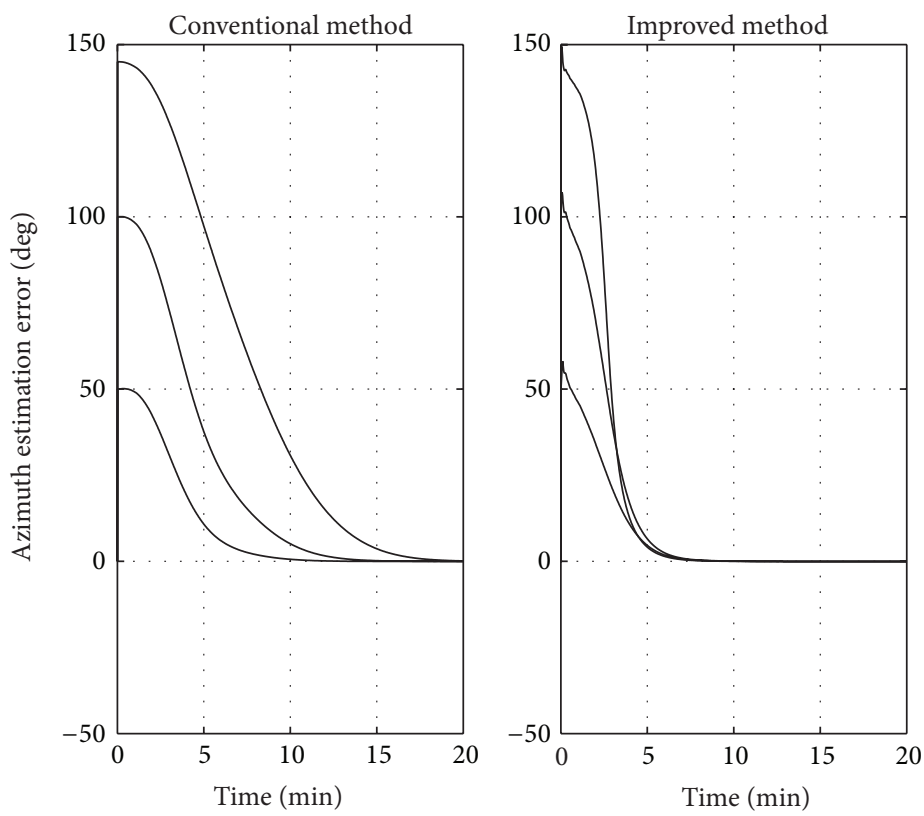

FIGURE 7: Gyrocompass alignment for $\psi_{U}=\left\{50^{\circ}, 100^{\circ}, 145^{\circ}\right\}$.

The constant accelerometer errors: $\nabla^{b}=$ $\left.\left[\begin{array}{lll}10^{-4} \mathrm{~g} & 10^{-4} \mathrm{~g} & 10^{-4} \mathrm{~g}\end{array}\right]\right]^{T}$.

The acceleration due to gravity: $g=9.78049 \mathrm{~m} / \mathrm{s}^{2}$.

The local longitude and latitude: $\lambda=126.6705^{\circ}, \varphi=$ $45.7796^{\circ}$.

The control networks of improved method: $G(s)=$ $H(s)=\left(\xi \omega_{n}\right)^{2} /\left(s+\xi \omega_{n}\right)^{2}$.

The natural frequency of conventional and improved methods: $\omega_{n}=0.02 \mathrm{rad} / \mathrm{s}$.

Under the disturbing motions, the vehicle undertakes angular and lineal movements. In angular movement, the pitch $\theta_{x}$, the roll $\theta_{y}$, and the yaw $\theta_{z}$ are controlled as

$$
\begin{aligned}
& \theta_{x}=7^{\circ} \sin \left(\frac{2 \pi}{5} t+\frac{\pi}{3}\right), \\
& \theta_{y}=5^{\circ} \sin \left(\frac{2 \pi}{7} t+\frac{\pi}{6}\right), \\
& \theta_{z}=10^{\circ} \sin \left(\frac{2 \pi}{6} t+\frac{\pi}{4}\right) .
\end{aligned}
$$

In the lineal movement, the vehicle lineal movement velocities are taken as

$$
\begin{aligned}
& V_{x}=-0.3 \sin \left(\frac{2 \pi}{10} t\right) \mathrm{m} / \mathrm{s}^{2}, \\
& V_{y}=-0.2 \sin \left(\frac{2 \pi}{6} t\right) \mathrm{m} / \mathrm{s}^{2}, \\
& V_{z}=-0.4 \sin \left(\frac{2 \pi}{8} t\right) \mathrm{m} / \mathrm{s}^{2} .
\end{aligned}
$$

Figures 7 and 8 provide the azimuth estimation errors of conventional and improved methods under the conditions of $\psi_{U}=\left\{50^{\circ}, 100^{\circ}, 145^{\circ},-50^{\circ},-100^{\circ},-145^{\circ}\right\}$. It can be seen that azimuth alignment could be accomplished by both conventional and improved gyrocompass alignment methods. But, under the condition of large azimuth misalignment, the performance of conventional method will be poor along with the growth of $\left|\psi_{U}\right|$. The reason is that the difference between the actual and desired eigenvalues is increasing along with the growth of $\left|\psi_{U}\right|$.

Figure 9 shows convergence time for different azimuth misalignments with a converged azimuth estimation error of less than 1 degree. It is clear that large azimuth misalignment needs more time to converge under the conventional method. However, for the improved method, the change of the convergence time is not obvious. That means the performance of the improved method is not affected by the large azimuth misalignment. The reason is that the eigenvalues of improved method can be set as the desired values, without the influence of the nonlinear factors caused by the large azimuth misalignment. Moreover, it is obvious that the alignment speed of the improved method is faster than that of the conventional one.

4.2. Experimental Results and Analysis. The experiments were implemented in the lab, as the true yaw angle of the strapdown INS was known, so different $\psi_{U}$ could be exactly set. We fixed the strapdown INS on the SGT-3 threeaxis turntable to implement the alignment experiments. The strapdown INS and the turntable can be seen in Figure 10. At the start of each experiment, the turntable turned to a static position for 20 minutes, that is, pitch angle $0^{\circ}$, roll angle $0^{\circ}$, and yaw angle $135^{\circ}$. Two alignment methods were performed under the condition of, respectively, setting initial yaw angle as $185^{\circ}$ and $235^{\circ}$; namely, $\psi_{U}=\left\{50^{\circ}, 100^{\circ}\right\}$. 

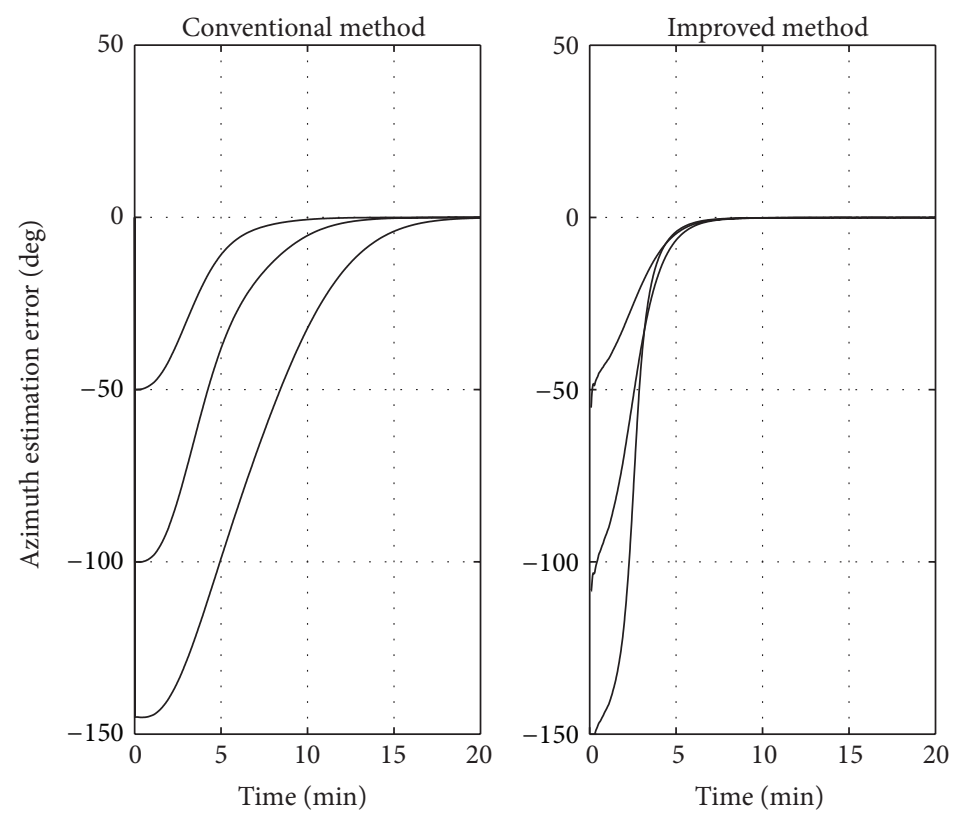

Figure 8: Gyrocompass alignment for $\psi_{U}=\left\{-50^{\circ},-100^{\circ},-145^{\circ}\right\}$.

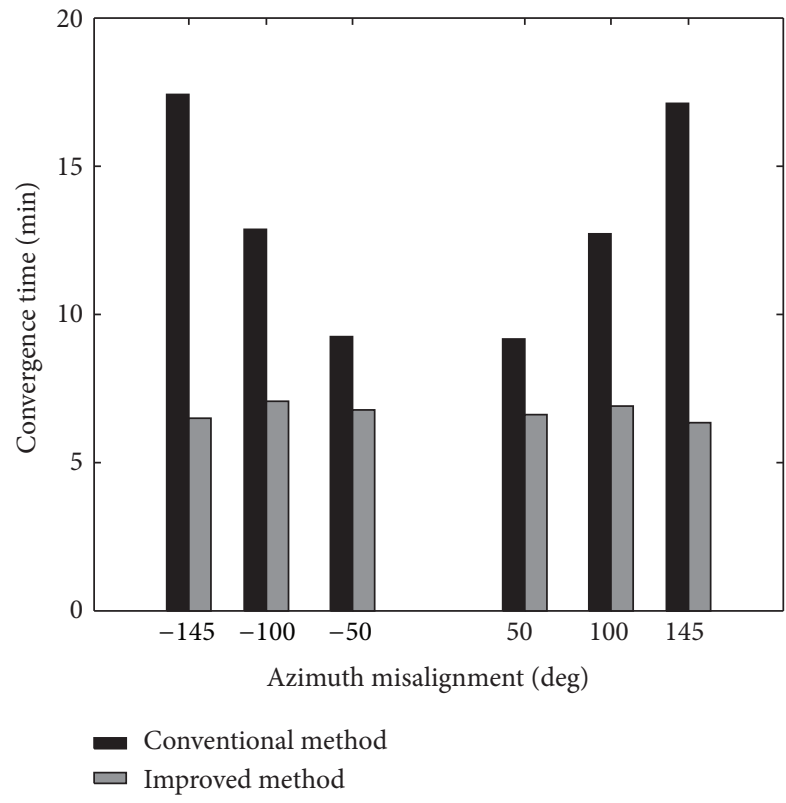

FIGURE 9: Convergence time comparison with different azimuth misalignments.

Figure 11 provides the comparison of the yaw angle using the two alignment methods under the condition of different $\psi_{U}$.

As the results above show, the yaw angle can converge to $135^{\circ}$ through using the two alignment methods. However, for the conventional method, the convergence time is lengthened with the growth of the azimuth misalignment. Compared with the conventional method, the convergence

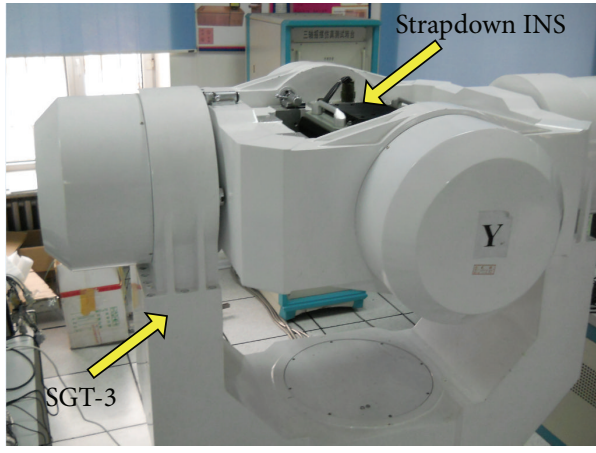

FIGURE 10: The SGT-3 turntable and strapdown INS.

time of improved method is hardly changed. The experimental results are consistent with the simulation results. The experiments demonstrate that the performance of the improved method is better than that of the conventional one.

\section{Conclusions}

In this paper, an improved gyrocompass alignment method has been proposed for large azimuth misalignment of INS. The improved new method is performed by opening the azimuth loop, and the estimation of the azimuth misalignment is implemented by using the horizontal velocity signals to estimate the nonlinear factors. Through using this method, the impact of the nonlinear factor caused by large azimuth misalignment on gyrocompass alignment is reduced, and the performance of gyrocompass alignment is improved. The alignment speed of the improved method is faster than the conventional one in the same conditions. The performance 


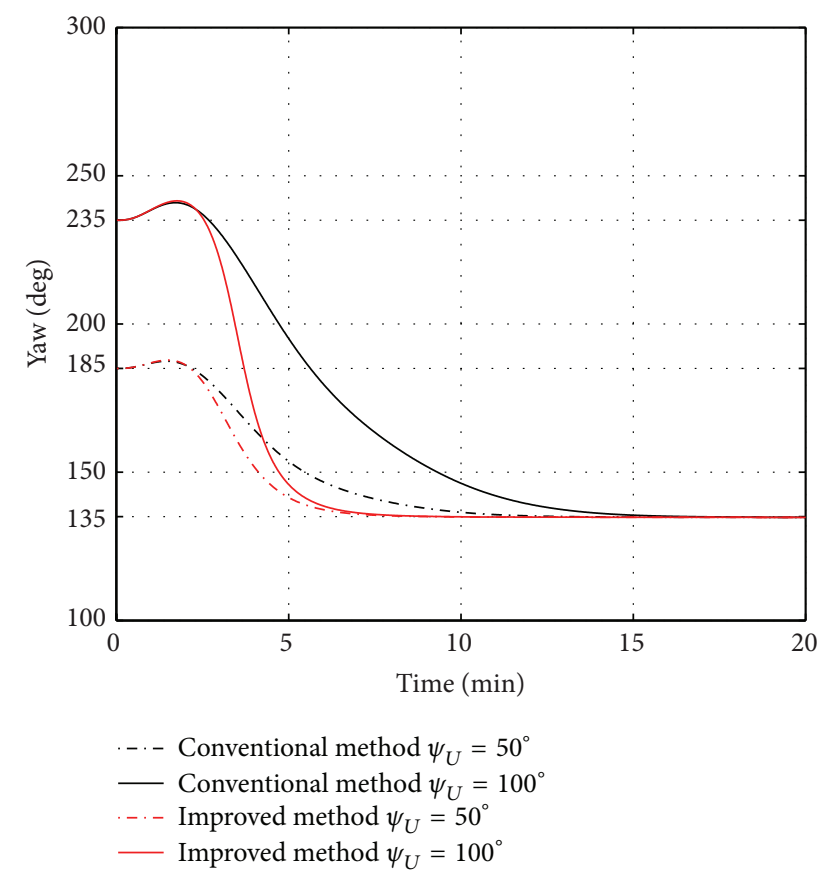

Figure 11: Comparison of the yaw angle using the two alignment methods.

of the improved method is well validated by simulations and experiments.

\section{Appendices}

\section{A. The Derivations of (31)}

In this section, (31) are obtained in the following.

According to (30), their matrix form can be written as

$$
\mathbf{A X}=\mathbf{B}
$$

where

A

$$
=\left[\begin{array}{cccc}
\frac{2 \xi \Omega \cos \varphi}{\omega_{n}} & 0 & 1 & -\frac{2 \xi \Omega \sin \varphi}{\omega_{n}} \\
\frac{g \Omega \cos \varphi}{\omega_{n}^{2}} & 0 & 0 & -\frac{g \Omega \sin \varphi}{\omega_{n}^{2}} \\
0 & \frac{2 \xi \Omega \cos \varphi}{\omega_{n}} & \frac{2 \xi \Omega \sin \varphi}{\omega_{n}} & 1 \\
0 & \frac{g \Omega \cos \varphi}{\omega_{n}^{2}} & \frac{g \Omega \sin \varphi}{\omega_{n}^{2}} & 0
\end{array}\right],
$$

$$
\begin{aligned}
\mathbf{X} & =\left[\begin{array}{c}
\sin \psi_{U} \\
\cos \psi_{U} \\
\psi_{E s s} \\
\psi_{N s s}
\end{array}\right], \\
\mathbf{B} & =\left[\begin{array}{c}
-\frac{\left(\mathbf{C}_{b}^{p} \nabla^{b}\right)_{N}}{g}-\frac{2 \xi}{\omega_{n}}\left(\mathbf{C}_{b}^{p} \boldsymbol{\varepsilon}^{b}\right)_{E} \\
-S\left(\psi_{U}\right)_{s s}-\frac{g}{\omega_{n}^{2}}\left(\mathbf{C}_{b}^{p} \boldsymbol{\varepsilon}^{b}\right)_{E} \\
\frac{2 \xi}{\omega_{n}} \cos \varphi+\frac{\left(\mathbf{C}_{b}^{p} \nabla^{b}\right)_{E}}{g}-\frac{2 \xi}{\omega_{n}}\left(\mathbf{C}_{b}^{p} \boldsymbol{\varepsilon}^{b}\right)_{N} \\
\frac{g}{\omega_{n}^{2}} \Omega \cos \varphi+C\left(\psi_{U}\right)_{s s}-\frac{g}{\omega_{n}^{2}}\left(\mathbf{C}_{b}^{p} \boldsymbol{\varepsilon}^{b}\right)_{N}
\end{array}\right] .
\end{aligned}
$$

Multiply the first and third rows of (A.1) by $\omega_{n} / 2 \xi \Omega \cos \varphi$, and multiply the second and fourth rows of (A.1) by $\omega_{n}^{2} / g \Omega \cos \varphi$; we can obtain

$$
\mathbf{A}_{1} \mathbf{X}=\mathbf{B}_{1}
$$

where

$$
\mathbf{A}_{1}=\left[\begin{array}{cccc}
1 & 0 & \frac{\omega_{n}}{2 \xi \Omega \cos \varphi} & -\tan \varphi \\
1 & 0 & 0 & -\tan \varphi \\
0 & 1 & \tan \varphi & \frac{\omega_{n}}{2 \xi \Omega \cos \varphi} \\
0 & 1 & \tan \varphi & 0
\end{array}\right]
$$

$$
\mathbf{X}=\left[\begin{array}{c}
\sin \psi_{U} \\
\cos \psi_{U} \\
\psi_{E s s} \\
\psi_{N s s}
\end{array}\right] \text {, }
$$

$$
\mathbf{B}_{1}=\left[\begin{array}{c}
-\frac{\omega_{n}}{2 \xi \Omega \cos \varphi} \frac{\left(\mathbf{C}_{b}^{p} \nabla^{b}\right)_{N}}{g}-\frac{\left(\mathbf{C}_{b}^{p} \boldsymbol{\varepsilon}^{b}\right)_{E}}{\Omega \cos \varphi} \\
-\frac{\omega_{n}^{2}}{g \Omega \cos \varphi} S\left(\psi_{U}\right)_{s s}-\frac{\left(\mathbf{C}_{b}^{p} \boldsymbol{\varepsilon}^{b}\right)_{E}}{\Omega \cos \varphi} \\
1+\frac{\omega_{n}}{2 \xi \Omega \cos \varphi} \frac{\left(\mathbf{C}_{b}^{p} \nabla^{b}\right)_{E}}{g}-\frac{\left(\mathbf{C}_{b}^{p} \boldsymbol{\varepsilon}^{b}\right)_{N}}{\Omega \cos \varphi} \\
1+\frac{\omega_{n}^{2}}{g \Omega \cos \varphi} C\left(\psi_{U}\right)_{\mathrm{ss}}-\frac{\left(\mathbf{C}_{b}^{p} \boldsymbol{\varepsilon}^{b}\right)_{N}}{\Omega \cos \varphi}
\end{array}\right] .
$$

Furthermore, by utilizing the elementary row transformation, (A.5) can be transformed into

$$
\mathbf{A}_{2} \mathbf{X}=\mathbf{B}_{2}
$$


where

$$
\begin{aligned}
& \mathbf{A}_{1}=\left[\begin{array}{cccc}
1 & 0 & 0 & -\tan \varphi \\
0 & 0 & -\frac{\omega_{n}}{2 \xi \Omega \cos \varphi} & 0 \\
0 & 1 & \tan \varphi & 0 \\
0 & 0 & 0 & -\frac{\omega_{n}}{2 \xi \Omega \cos \varphi}
\end{array}\right], \\
& \mathbf{X}=\left[\begin{array}{c}
\sin \psi_{U} \\
\cos \psi_{U} \\
\psi_{E s s} \\
\psi_{N s s}
\end{array}\right] \text {, } \\
& \text { B }_{1} \\
& {\left[\begin{array}{c}
-\frac{\omega_{n}^{2}}{g \Omega \cos \varphi} S\left(\psi_{U}\right)_{\mathrm{ss}}-\frac{\left(\mathbf{C}_{b}^{p} \boldsymbol{\varepsilon}^{b}\right)_{E}}{\Omega \cos \varphi} \\
-\frac{\omega_{n}^{2}}{g \Omega \cos \varphi} S\left(\psi_{U}\right)_{\mathrm{ss}}-\frac{\omega_{n}}{2 \xi \Omega \cos \varphi} \frac{\left(\mathbf{C}_{b}^{p} \nabla^{b}\right)_{N}}{g} \\
1+\frac{\omega_{n}^{2}}{g \Omega \cos \varphi} C\left(\psi_{U}\right)_{\mathrm{ss}}-\frac{\left(\mathbf{C}_{b}^{p} \boldsymbol{\varepsilon}^{b}\right)_{N}}{\Omega \cos \varphi} \\
\frac{\omega_{n}^{2}}{g \Omega \cos \varphi} C\left(\psi_{U}\right)_{\mathrm{ss}}-\frac{\omega_{n}}{2 \xi \Omega \cos \varphi} \frac{\left(\mathbf{C}_{b}^{p} \nabla^{b}\right)_{E}}{g}
\end{array}\right] .}
\end{aligned}
$$

Then, according to matrix equation (A.7), we have

$$
\begin{aligned}
\sin \psi_{U}= & -\frac{\omega_{n}^{2}}{g \Omega \cos \varphi} \cdot S\left(\psi_{U}\right)_{\mathrm{ss}}-\frac{2 \xi \omega_{n} \tan \varphi}{g} \\
& \cdot C\left(\psi_{U}\right)_{\mathrm{ss}}-\frac{\left(\mathbf{C}_{b}^{p} \boldsymbol{\varepsilon}^{b}\right)_{E}}{\Omega \cos \varphi} \\
& +\frac{\left(\mathbf{C}_{b}^{p} \nabla^{b}\right)_{E}}{g} \tan \varphi, \\
\cos \psi_{U}= & +\frac{\omega_{n}^{2}}{g \Omega \cos \varphi} \cdot C\left(\psi_{U}\right)_{\mathrm{ss}}-\frac{2 \xi \omega_{n} \tan \varphi}{g} \\
& \cdot S\left(\psi_{U}\right)_{\mathrm{ss}}-\frac{\left(\mathbf{C}_{b}^{p} \boldsymbol{\varepsilon}^{b}\right)_{N}}{\Omega \cos \varphi} \\
& +\frac{\left(\mathbf{C}_{b}^{p} \nabla^{b}\right)_{N}}{g} \tan \varphi .
\end{aligned}
$$

\section{B. The Derivations of (34)}

The derivations of (34) are carried out based on the principle of least squares fitting, setting the Euclidean minimum norm $J$ between $\sin \widetilde{\psi}_{U}, \cos \widetilde{\psi}_{U}$ and $\sin \widehat{\psi}_{U}, \cos \widehat{\psi}_{U}$ (the normalized data) as the normalization index.

First, define

$$
J=\left[\left(\sin \widetilde{\psi}_{U}-\sin \widehat{\psi}_{U}\right)^{2}+\left(\cos \widetilde{\psi}_{U}-\cos \widehat{\psi}_{U}\right)^{2}\right]^{1 / 2} .
$$

It is subjected to the constrained condition; namely,

$$
h=\left(\sin \widehat{\psi}_{U}\right)^{2}+\left(\cos \widehat{\psi}_{U}\right)^{2}-1=0 .
$$

Second, define an auxiliary function $m$; namely,

$$
m=J^{2}+\lambda h,
$$

where $\lambda$ is an unknown parameter.

By substituting (B.1) and (B.2) into (B.3), we can get

$$
\begin{aligned}
m=[ & {\left[\left(\sin \widetilde{\psi}_{U}-\sin \widehat{\psi}_{U}\right)^{2}+\left(\cos \widetilde{\psi}_{U}-\cos \widehat{\psi}_{U}\right)^{2}\right] } \\
& -\lambda\left(\left(\sin \widehat{\psi}_{U}\right)^{2}+\left(\cos \widehat{\psi}_{U}\right)^{2}-1\right) .
\end{aligned}
$$

Obviously, the parameters $\sin \widehat{\psi}_{U}$ and $\cos \widehat{\psi}_{U}$ that made $J$ minimum are equal to the ones that make $m$ minimum. Respectively, the partial derivatives of the parameters $\sin \widehat{\psi}_{U}$ and $\cos \widehat{\psi}_{U}$ are calculated, and, by making them equal to zero, we can get

$$
\begin{aligned}
& \sin \widehat{\psi}_{U}=\frac{\sin \widetilde{\psi}_{U}}{1+\lambda}, \\
& \cos \widehat{\psi}_{U}=\frac{\cos \widetilde{\psi}_{U}}{1+\lambda} .
\end{aligned}
$$

Then, by substituting (B.5) into (B.2), we have

$$
\frac{\left(\sin \widetilde{\psi}_{U}\right)^{2}+\left(\cos \widetilde{\psi}_{U}\right)^{2}}{(1+\lambda)^{2}}=1 .
$$

Finally, combining (B.5) and (B.6), the normalization equations can be obtained:

$$
\begin{aligned}
& \sin \widehat{\psi}_{U}=\frac{\sin \widetilde{\psi}_{U}}{\sqrt{\sin ^{2} \widetilde{\psi}_{U}+\cos ^{2} \widetilde{\psi}_{U}}}, \\
& \cos \widehat{\psi}_{U}=\frac{\cos \widetilde{\psi}_{U}}{\sqrt{\sin ^{2} \widetilde{\psi}_{U}+\cos ^{2} \tilde{\psi}_{U}}} .
\end{aligned}
$$

\section{Conflict of Interests}

The authors declare that there is no conflict of interests regarding the publication of this paper.

\section{Acknowledgments}

The work described in the paper was supported by the National Natural Science Foundation of China (61203225) and the National Science Foundation for Post-Doctoral Scientists of China (2012M510083). The authors would like to thank all members of the Inertial Navigation Research Group at Harbin Engineering University for the technical assistance with the navigation system.

\section{References}

[1] H. W. Park, J. G. Lee, and C. G. Park, "Covariance analysis of strapdown INS considering gyrocompass characteristics," IEEE Transactions on Aerospace and Electronic Systems, vol. 31, no. 1, pp. 320-328, 1995. 
[2] R. A. Nash Jr., J. A. D’Appolito, and K. J. Roy, "Error analysis of hybrid aircraft inertial navigation systems," in Proceedings of the AIAA Guidance and Control Conference, Stanford, Calif, USA, 1972.

[3] F. D. Jurenka and C. T. Leondes, "Optimum alignment of an inertial autonavigator," IEEE Transactions on Aerospace and Electronic Systems, vol. AES-3, no. 6, pp. 880-888, 1967.

[4] R. H. Cannon, "Alignment of inertial guidance systems by gyrocompassing-linear theory," Journal of Aerospace Science, vol. 28, no. 11, pp. 885-895, 912, 1961.

[5] G. R. Pitman Jr., Inertial Guidance, John Wiley \& Sons, New York, NY, USA, 1962.

[6] K. R. Britting, Inertial Navigation Systems Analysis, John Wiley \& Sons, New York, NY, USA, 1971.

[7] C. San Giovanni Jr. and E. Levinson, "Performance of a ring laser strapdown marine gyrocompass," Navigation, vol. 28, no. 4, pp. 311-341, 1982.

[8] X. Liu, X. Xu, Y. Liu, and L. Wang, "A fast and high-accuracy compass alignment method to SINS with azimuth axis rotation," Mathematical Problems in Engineering, vol. 2013, Article ID 524284, 12 pages, 2013.

[9] F. Sun, Q. Wang, Z. Qi, and C. Wang, "Research on the estimation method of DVL velocity error based on double program in Fiber Optic Gyro SINS," Optik, vol. 124, no. 22, pp. 5344-5349, 2013.

[10] B. M. Scherzinger, "Inertial navigator error models for large heading uncertainty," in Proceedings of the IEEE Position Location and Navigation Symposium (PLANS '96), pp. 477-484, Atlanta, Ga, USA, April 1996.

[11] X. Y. Kong, E. M. Nebot, and H. Durrant-Whyte, "Development of a non-linear psi-angle model for large misalignment errors and its application in INS alignment and calibration," in Proceedings of the 1999 IEEE International Conference on Robotics \& Automation (ICRA '99), pp. 1430-1435, Detroit, Mich, USA, May 1999.

[12] M.-J. Yu, H.-W. Park, and C.-B. Jeon, "Equivalent nonlinear error models of strapdown inertial navigation system," in Proceedings of the Guidance, Navigation, and Control Conference, AIAA-97-3563, pp. 581-587, 1997.

[13] T. Abbas, Z. Yunyan, and L. Yanjun, "SINS initial alignment for small tilt and large azimuth misalignment angles," in Proceedings of the IEEE 3rd International Conference on Communication Software and Networks (ICCSN '11), pp. 628-632, Xian, China, May 2011.

[14] S. L. Han and J. L. Wang, "A novel initial alignment scheme for low-cost INS aided by GPS for land vehicle applications," The Journal of Navigation, vol. 63, no. 4, pp. 663-680, 2010.

[15] P. G. Savage, Strapdown Analytics, Strapdown Associates, 2nd edition, 2007.

[16] I. Yaesh and B. Priel, "Design of leveling loop for marine navigation system," IEEE Transactions on Aerospace and Electronic Systems, vol. 29, no. 2, pp. 599-604, 1993. 


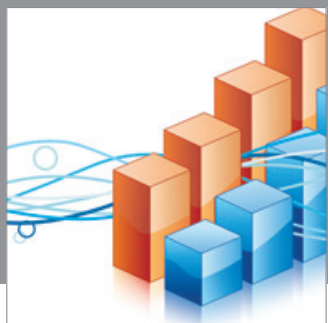

Advances in

Operations Research

mansans

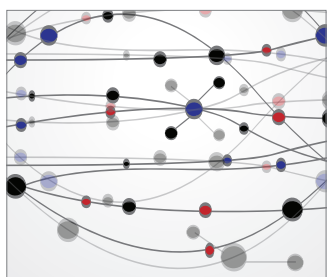

The Scientific World Journal
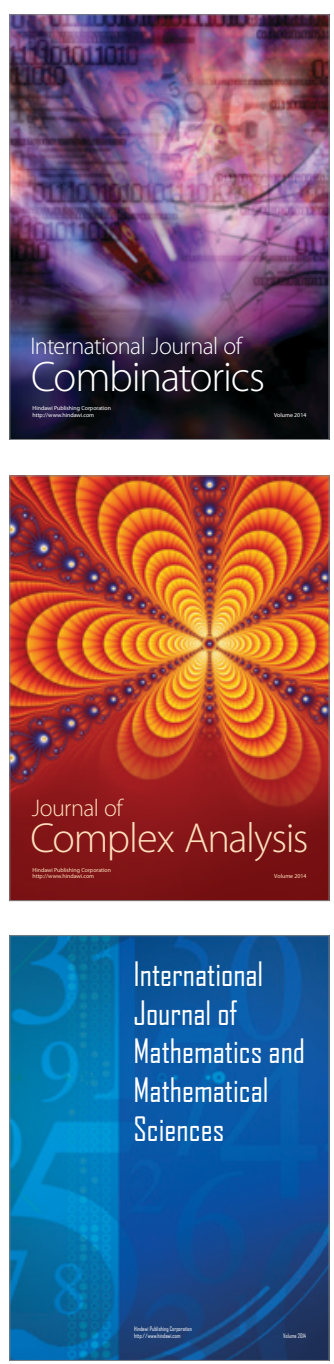
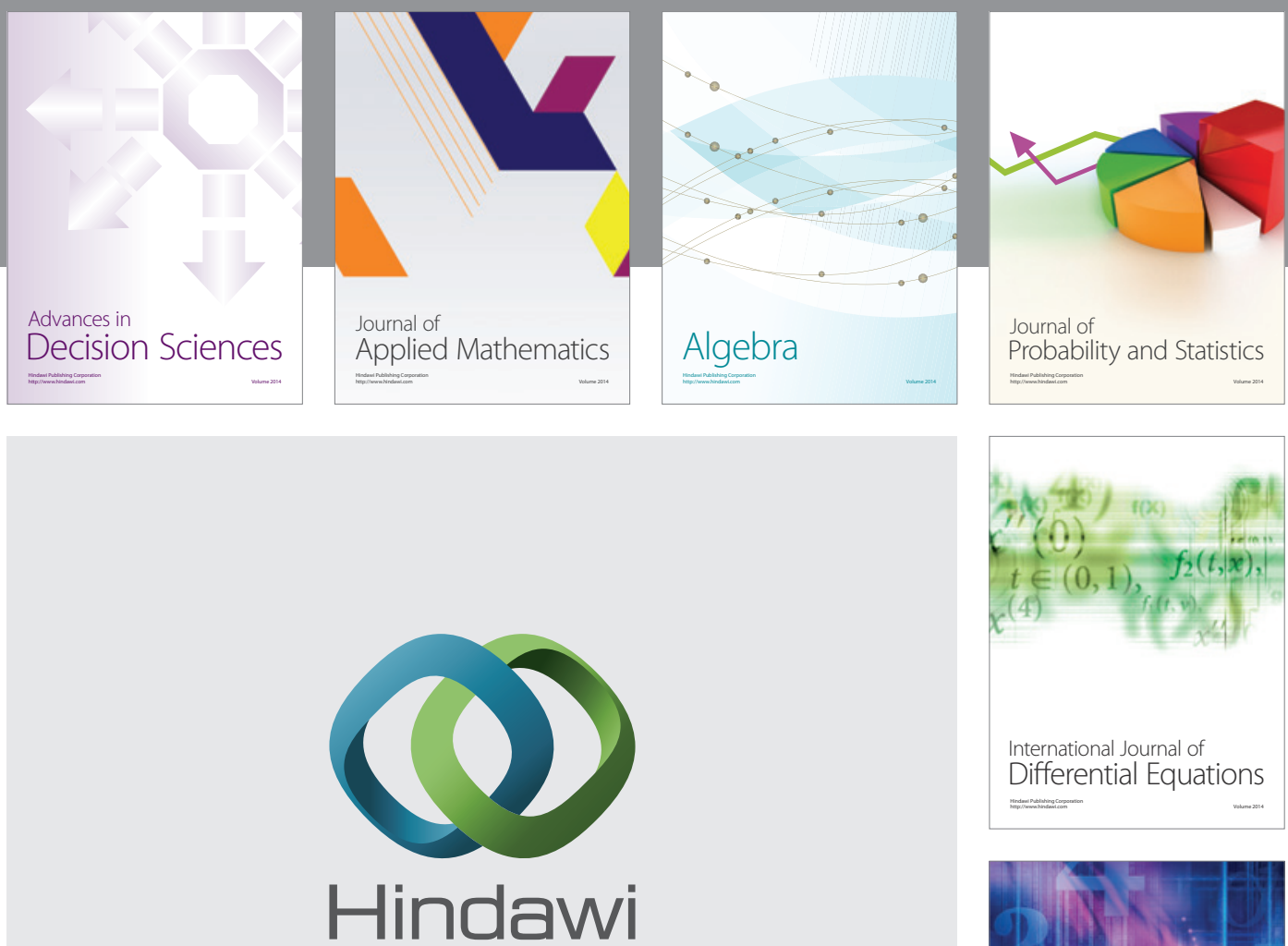

Submit your manuscripts at http://www.hindawi.com
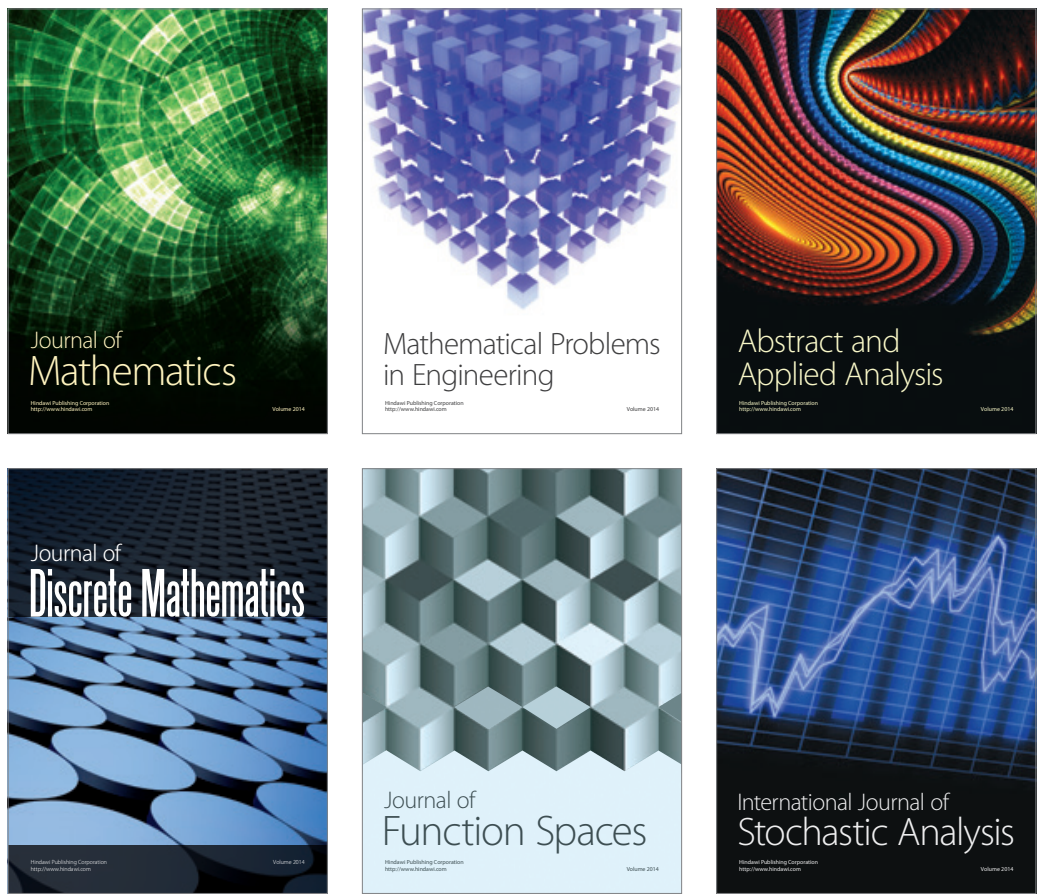

Journal of

Function Spaces

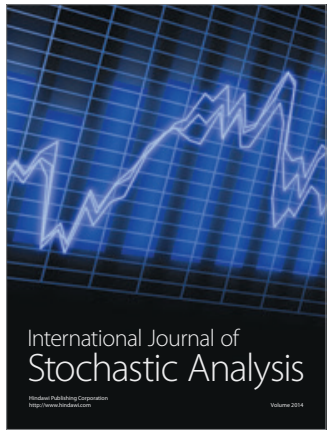

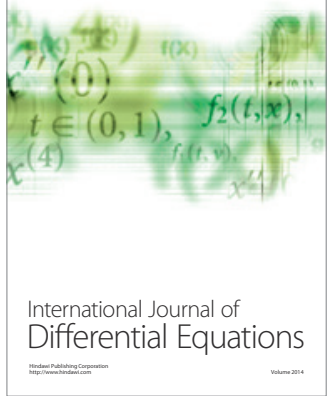
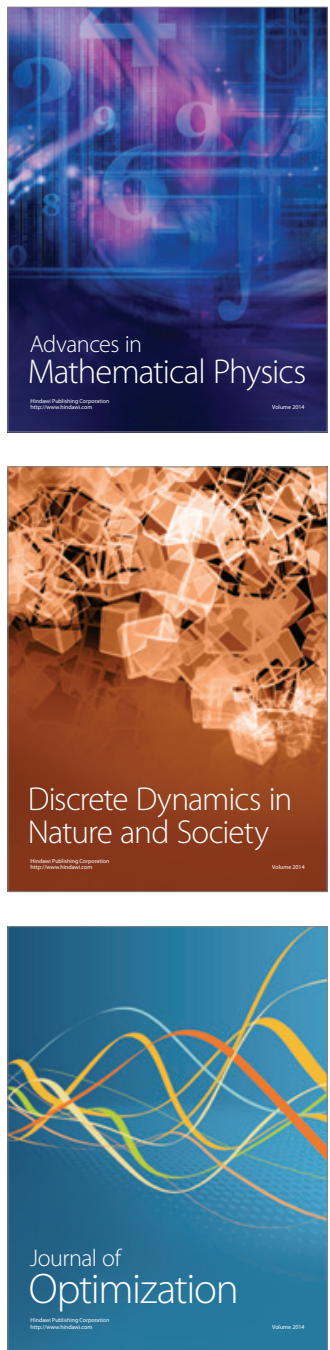